\title{
RADIATION-ACCELLERATED IONS IN HOT STAR WINDS: HEATING AND “TURBULENCE" EFFECTS
}

\author{
E.YA. VILKOVISKIJ \\ V.G. Fessenkov Astrophysical Institute of the National Academy of Sciences, 480068 \\ Almaty, Observatory AFI, Kazakhstan
}

In the papers by Vilkoviskij (1981), Vilkoviskij and Tambovtzeva (1988) and Springmann and Pauldrach (1992) it was shown that ions can reach velocities $v_{i} \geq \bar{v}_{T p}\left(\bar{v}_{T p}=\left(2 k T / m_{p}\right)^{1 / 2}\right)$ relative to the wind plasma when the radiation pressure force exceeds the protons frictional force. The condition for this transition can be estimated as

$$
v_{7} \geq 0.7 \dot{M}_{6} z_{i}^{2} /\left(R_{1}^{2} T_{4} \phi\left(\lambda_{i} T_{*}\right)\right),
$$

where $v_{7}$ is the wind velocity in units of $10^{7} \mathrm{~cm} / \mathrm{s}, \dot{M}_{6}=d M / d t$ the stellar mass-loss rate in units of $10^{-6} M_{\odot} / \mathrm{yr}, z_{i}$ the ion charge, $R_{1}=R_{*} /\left(10 R_{\odot}\right)$, $\phi\left(\lambda_{i} T_{*}\right)=f_{i} \lambda_{5}^{-3} / e^{14.4 /\left(\lambda_{5} T_{4}\right)-1}, \lambda_{5}=\lambda_{i} / 10^{3} \AA$ and $T_{4}=T_{e} / 10^{4} \mathrm{~K}$. The ion distribution function consists of two parts: the part $D F_{1}$ approximates the "shifted Maxwell" distribution, and the non-Maxwellian part $D F_{2}$ is for "runaway" particles:

$$
D F\left(v_{i}\right)=\left(1-q_{2}\right) D F_{1 i}+q_{2} D F_{2 i},
$$

where $q_{2}>0$ when condition (1) is fulfilled. The specific heat power, divided by $N_{p}^{2}$ is

$$
H=N_{i} / N_{p}^{2} \int_{0}^{\infty} v_{i} D F\left(v_{i}\right) F_{i p} d v_{i},
$$

where $N_{i}$ and $N_{p}$ are the ion and proton densities and $F_{i p}$ is the frictional force. If the "runaway condition" (1) is not fulfilled, the mean velocity of ions relative to the wind plasma $\bar{v}_{i}$ is less then $\bar{v}_{T p}$, but it rises to about the electron thermal velocity $\bar{v}_{e p} \sim 43 \bar{v}_{T p}$ in the opposite case.

So we can estimate

$$
H=H^{m}\left(\bar{v}_{i} / v_{T p}\left(1-q_{2}\right)+43 q_{2}\right),
$$

where $H^{m} \cong \pi e^{4} z_{i}^{2} N_{i}\left(N_{p} k T_{p}\right)^{-1} \ln \Lambda v_{T p}=\left(N_{i}\right)$

which is $\sim 2 \cdot 10^{-22} z_{i}^{2} T_{p 4}^{-1 / 2}\left(n_{i} / 10^{-4}\right) \mathrm{erg} \mathrm{cm}^{3} / \mathrm{s}$, where $n_{i}=N_{i} / N_{p}$.

Model calculations show that for the O-and early B-type stars this "kinetic heat" is sufficient for heating of the wind to a temperature $T_{e} \sim 10^{5} \mathrm{~K}$ at $R \geq 2 R_{*}$ and to $T_{e} \geq 10^{6} \mathrm{~K}$ at $R \geq 100 R_{*}$, and the ion's velocity distribution can manifest itself as "turbulence" in spectral lines. 
So the physical picture including kinetic heat resembles the empirical "warm wind" model, and moreover, it predicts an outer hot corona at $R \geq$ $R_{k} \sim 100 R_{*}$. The X-ray luminosity of the corona is $L_{x}=4 \pi \int_{R k}^{\infty} \epsilon_{X} R^{2} d R$. With $\dot{M}=4 \pi \rho v r^{2}, \epsilon_{X} \cong 2 \cdot 10^{-27} N_{e}^{2} T_{e}^{1 / 2} \mathrm{erg} / \mathrm{cm}^{3} \mathrm{~s}$, we have

$$
L_{x} \cong 5 \cdot 10^{32} T_{e 7}^{1 / 2} \dot{M}_{6}^{2} /\left(R_{1} v_{8}^{2}\left(r_{k} / 100\right)\right) \mathrm{erg} / \mathrm{s}
$$

where $R_{1}$ is the stellar radius in units of $10 R_{\odot}, r_{k}=R_{k} / R_{*}, T_{e 7}=T_{e} / 10^{7} \mathrm{~K}$ and $v_{8}$ is the wind velocity in units of $10^{3} \mathrm{~km} / \mathrm{s}$ at $R_{k}$.

We can predict the wide dispersion of X-ray luminosities as due to the dispersion in stellar parameters, and the X-ray variability (with a characteristic time scale of several hours to days) as due to stellar wind variability.

\section{References}

Springmann U.V.E., Pauldrach A.W.A.: 1992, Astronomy and Astrophysics 262, 515 Vilkoviskij E.Ya.: 1981, Astrofisica (Sov) 17, 310

Vilkoviskij E.Ya., Tambovtzeva L.V.: 1988, in: "Mass Outflows", Bianchi L., Gilmozzi R. (eds.), p. 195 